\title{
References
}

[1] S. B ourne, The Jacobson radical of a semiring, Proc. Nat. Acad. Sci. 37 (1951), pp. 163-170.

[2] - and. H. Zassenhaus, On the semiradical of a semiring, Proc. Nat. Acad. Sci. 44 (1958), pp. 907-914.

[3] G. Birkhoff, Lattice theory, rev. ed., Colloquium Publication No. 25, Amer. Math. Soc., New York 1948.

[4] - and R. S. Pierce, Lattice-ordered rings, An. Acad. Brasil. Ci. 28 (1956), pp. $41-69$.

[5] L. Fuchs, Partially ordered algebraic systems, International series on pure and applied mathematics, Vol. 28, Oxford 1963.

[6] M. Henriksen, Ideals in semirings with commutative addition, Notices Amer. Math. Soc. 6 (1958), p. 321.

[7] W. Słowikowski and W. Zawadowski, A generalization of maximal ideal methods of Stone and Gelfond, Fund. Math. 42 (1955), pp. 215-231.

Resu par la Rédaction le 4.6.1965

\section{Regular iteration of functions with multiplier 1}

\section{by \\ A. Smajdor (Katowice)}

Let $f(x)$ be a function which is defined, continuous and strictly increasing in an interval $(a, b), a \geqslant-\infty, b \leqslant+\infty$. Furthermore

$$
a<f(x)<x \text { for } \quad x \in(a, b) \text {. }
$$

Definitron 1. A one-parametr family of functions $f^{u}(x), u \in(-\infty$, $+\infty)$, is called an iteration group of the function $f(x)$ provided that the following conditions are fulfilled (see [6], [4]):

(I) for every $u \in(-\infty,+\infty)$ the function $f^{u}(x)$ is defined, continuous and strictly increasing in an interval $\left(a, b_{u}\right)$, where $a<b_{u} \leqslant b$;

(II) for every pair of $u, v \in(-\infty,+\infty)$

$$
f^{u}\left[f^{v}(x)\right]=f^{u+v}(x)
$$

holds for every $x$ for which both sides are meaningful;

(III) $f^{1}(x)=f(x)$ for $x \in(a, b)$;

(IV) for every fixed $x \in(a, b), f^{u}(x)$ is a oontinuous function of $u$.

It follows from conditions (II) and (III) that, for integral $u, f^{u}(x)$ are identical with the natural iterates $f^{n}(x)$ of the function $f(x)$ defined by

$$
f^{0}(x)=x, \quad f^{n+1}(x)=f\left[f^{n}(x)\right], \quad n=0, \pm 1, \pm 2, \ldots
$$

We note also that it follows from (II) that for every fixed iteration group and for every $u$ and $v$ the functions $f^{u}(x)$ and $f^{v}(x)$ are commutative:

$$
f^{u}\left[f^{v}(x)\right]=f^{v}\left[f^{u}(x)\right]
$$

It is known (see [1], [2], [9]) that every iteration group $f^{u}(x)$ of the function $f(x)$ is given by the formula

$$
f^{u}(x)=\alpha^{-1}[\alpha(x)+u],
$$

where $\alpha(x)$ is a continuous and strictly monotonic solution of the Abel equation
(3)
$\alpha[f(x)]=a(x)+1$,

Fundamenta Mathematicae, T. LIX 
and conversely, for every continuous and strictly monotonic solution of equation (3), formula (2) defines an iteration group $f^{u}(x)$ of the function $f(x)$.

It is also known (see [3]) that a continuous and strictly monotonic solution of equation (3) may by almost arbitrarily prescribed in an interval $[f(\xi), \xi]$. Hence it follows that there exist infinitely many different iteration groups of the function $f(x)$ (see also [6]).

In the sequel we shall assume that the function $f(x)$ fulfils the condition.

$$
\lim _{x \rightarrow a+} \frac{f(x)-a^{*}}{x-a^{*}}=1
$$

where $a^{*}=a$ if $a>-\infty, a^{*}=0$ if $a=-\infty$.

We accept after Szekeres ([7], [8]) the following definition.

DefinItion 2. An iteration group of the function $f(x)$ is called regular whenever

$$
\lim _{x \rightarrow a+} \frac{x-f^{u}(x)}{x-f(x)}=u
$$

for every $u \in(-\infty,+\infty)$.

Let $\xi$ be an arbitrarily fixed point of the interval $(a, b)$. If the limit

$$
\alpha(x)=\lim _{n \rightarrow \infty} \frac{f^{n}(x)-f^{n}(\xi)}{f^{n+1}(\xi)-f^{n}(\xi)}
$$

exists for every $x \in(a, b)$ and satisfies equation (3) in the interval $(a, b)$, then $\alpha(x)$ is called the principal solution of equation (3). The principal solution is unique up to an additive constant and changing $\xi$ in formula (5) we change the function $\alpha(x)$ by an additive constant.

DEFinimion 3. If the function $\alpha(x)$ is a continuous and strictly increasing principal solution of equation (3), then the iteration group of the function $f(x)$, defined by formula (2), is called the principal iteration group of the function $f(x)$.

Let us notice that though the principal solution of equation (3) is defined up to an additive constant, nevertheless the principal iteration group is unique.

The purpose of the present paper is to prove the equivalence of the notions of the regular iteration group and the principal iteration group. The idea of the proof is similar to that of an analogous theorem in a paper by $\mathbf{A}$. Lundberg [5], concerning functions with multipliers different from 0 and 1. (We give the term multiplier to the number $s=\lim _{x \rightarrow a+} \frac{a^{*}-f(x)}{a^{*}-x}$.) Analogous results have been obtained recently on other lines by R. Coifman (C. R. Paris 258 (1964), pp. 1976-1977).
THEOREM 1. The principal iteration group is regular.

Proof. We assume that the iteration group $f^{u}(x)$ of the function $f(x)$ is the principal iteration group, i.e. $f^{u}(x)=a^{-1}[\alpha(x)+u]$, where $\alpha(x)$ is a continuous and strictly monotonic solution of equation (3) of form (5). The sequences

$$
\frac{f^{n}(x)-f^{n}(\xi)}{f^{n+1}(\xi)-f^{n}(\xi)}, \quad \frac{f^{n+1}(x)-f^{n}(\xi)}{f^{n+1}(\xi)-f^{n}(\xi)}
$$

are sequences of increasing functions in the interval $(a, b)$, convergent to a continuous limit function. Therefore they are uniformly convergent to the functions $\alpha(x)$ and $\alpha[f(x)]$, respectively, in every interval $\left[a_{1}, b_{1}\right]$ $\widetilde{C}(a, b)$. Hence on every rectangle $\left[a_{1}, b_{1}\right] \times\left[a_{2}, b_{2}\right] \subset(a, b) \times(a, b)$ the sequence

$$
\frac{f^{n}(x)-f^{n}(y)}{f^{n+1}(\xi)-f^{n}(\xi)}=\frac{f^{n}(x)-f^{n}(\xi)}{f^{n+1}(\xi)-f^{n}(\xi)}-\frac{f^{n}(y)-f^{n}(\xi)}{f^{n+1}(\xi)-f^{n}(\xi)}
$$

is uniformly convergent to $\alpha(x)-a(y)$ and the sequence

$$
\frac{f^{n+1}(y)-f^{n}(y)}{f^{n+1}(\xi)-f^{n}(\xi)}=\frac{f^{n+1}(y)-f^{n}(\xi)}{f^{n+1}(\xi)-f^{n}(\xi)}-\frac{f^{n}(y)-f^{n}(\xi)}{f^{n+1}(\xi)-f^{n}(\xi)}
$$

is uniformly convergent in every interval $\left[a_{2}, b_{2}\right] \subset(a, b)$ to the function $\alpha[f(y)]-\alpha(y)=1$ (cf. (3)). Thus the sequence

$$
\frac{f^{n}(x)-f^{n}(y)}{f^{n+1}(y)-f^{n}(y)}=\frac{f^{n}(x)-f^{n}(y)}{f^{n+1}(\xi)-f^{n}(\xi)} \cdot \frac{f^{n+1}(\xi)-f^{n}(\xi)}{f^{n+1}(y)-f^{n}(y)}
$$

is uniformly convergent to $a(x)-a(y)$ in every rectangle $\left[a_{1}, b_{1}\right] \times\left[a_{2}, b_{2}\right]$ $C(a, b) \times(a, b)$. Thus, for every $\varepsilon>0$ there exists an $N$ such that

$$
\left|\frac{f^{n}(x)-f^{n}(y)}{f^{n+1}(y)-f^{n}(y)}-[a(x)-\alpha(y)]\right|<\varepsilon
$$

for every $n>N$ and $(x, y) \in\left[a_{1}, b_{1}\right] \times\left[a_{2}, b_{2}\right]$.

Let us fix a real number $u$. In order to prove (4) we shall show that for an arbitrary sequence $\left\{x_{m}\right\}$ such that $x_{m} \rightarrow a$ as $m \rightarrow \infty$ and $x_{m} \in(a, b)$ for $m=1,2, \ldots$ we have

$$
\lim _{m \rightarrow \infty} \frac{x_{m}-f^{u}\left(x_{m}\right)}{x_{m}-f\left(x_{m}\right)}=u
$$

It is sufficient to prove (7) when $\left\{x_{m}\right\}$ is a strictly decreasing sequence. For every $m$ there exists a positive integer $n_{m}$ such that

$$
f^{-n_{m}}\left(x_{m}\right) \in\left[f\left(x_{1}\right), x_{1}\right]
$$


The sequence $\left\{n_{m}\right\}$ is increasing and $n_{m} \rightarrow \infty$ as $m \rightarrow \infty$. Let

$$
t_{m}=f^{-n_{m}}\left(x_{m}\right) \text {. }
$$

From (I) (cf. also (2)) it follows that the function $f^{u}(x)$ is strictly increasing. Thus by (8) we obtain

$$
\left(f^{u}\left(t_{m}\right), t_{m}\right) \in\left[f^{u+1}\left(x_{1}\right), f^{u}\left(x_{1}\right)\right] \times\left[f\left(x_{1}\right), x_{1}\right] .
$$

Let

$$
a_{1}=f^{u+1}\left(x_{1}\right), \quad b_{1}=f^{u}\left(x_{1}\right), \quad a_{2}=f\left(x_{1}\right), \quad b_{2}=x_{1}
$$

then by (6) we have

$$
\left|\frac{f^{n_{m}}\left[f^{u}\left(t_{m}\right)\right]-f^{n_{m}}\left(t_{m}\right)}{f^{n_{m}+1}\left(t_{m}\right)-f^{n_{m}}\left(t_{m}\right)}-\left(\alpha\left[f^{u}\left(t_{m}\right)\right]-\alpha\left(t_{m}\right)\right)\right|<\varepsilon
$$

for $m>M$ (where $M$ is chosen so that $n_{m}>N$ for $m>M$ ). According to (1) and (9) we get

$$
\frac{f^{n_{m}}\left[f^{u}\left(t_{m}\right)\right]-f^{n_{m}}\left(t_{m}\right)}{f^{n_{m}+1}\left(t_{m}\right)-f^{n_{m}}\left(t_{m}\right)}=\frac{f^{u}\left[f^{n_{m}}\left(t_{m}\right)\right]-f^{n_{m}}\left(t_{m}\right)}{f\left[f^{n_{m}}\left(t_{m}\right)\right]-f^{n_{m}}\left(t_{m}\right)}=\frac{f^{u}\left(x_{m}\right)-x_{m}}{f\left(x_{m}\right)-x_{m}} .
$$

Since $f^{u}\left(t_{m}\right)=\alpha^{-1}\left[\alpha\left(t_{m}\right)+u\right]$, we have $\alpha\left[f^{u}\left(t_{m}\right)\right]-\alpha\left(t_{m}\right)=u$ and finally we get by (10)

$$
\left|\frac{x_{m}-f^{u}\left(x_{m}\right)}{x_{m}-f\left(x_{m}\right)}-u\right|<\varepsilon
$$

for $m>M$, which proves formula (7). Condition (4) follows from (7) in view of the fact that the sequence $\left\{x_{m}\right\}$ has been arbitrary. Thus the iteration group $f^{u}(x)$ of the function $f(x)$ is regular.

THEOREM 2. A regular iteration group is principal.

Proof. Let the family of functions $f^{u}(x)$ be the regular iteration group of the function $f(x)$. There exists a function $a(x)$ defined in $(a, b)$ continuous and strictly monotonic, fulfilling equation (3) and such that formula (2) holds. Moreover, condition (4) is fulfilled for every real number $u$. We shall show that the function $\alpha(x)$ is the principal solution of equation (3).

Let $x, \xi \in(a, b)$. Since in (2) we can always replace $\alpha(x)$ by $\alpha(x)-$ $-\alpha(\xi)$, in what follows we may assume without loss of generality that $a(\xi)=0$. By induction we get from (3)

\section{(11)}

whence

$$
\alpha\left[f^{n}(x)\right]=a(x)+n,
$$

and

(12)

$$
\alpha\left[f^{n}(\xi)\right]=n
$$

It follows from (12) that

$$
\lim _{n \rightarrow \infty} \alpha^{-1}(n)=0 .
$$

By (2) and (4) we have

$$
\lim _{t \rightarrow a+} \frac{t-a^{-1}[a(t)+u]}{t-f(t)}=u .
$$

Let us put in (14) $t=\alpha^{-1}(n)$ and $u=\alpha(x)$. On account of (13), we get

$$
\lim _{n \rightarrow \infty} \frac{\alpha^{-1}(n)-a^{-1}(n+\alpha(x))}{a^{-1}(n)-f\left[\alpha^{-1}(n)\right]}=\alpha(x)
$$

and in view of (11) and (12) we get

$$
\lim _{n \rightarrow \infty} \frac{f^{n}(x)-f^{n}(\xi)}{f^{n+1}(\xi)-f^{n}(\xi)}=\alpha(x)
$$

i.e. $\alpha(x)$ is the principal solution of equation (3). Thus the iteration group $f^{u}(x)$ of the function $f(x)$ generated by $\alpha(x)$ is the principal iteration group.

\section{References}

[1] J. Aczél, Vorlesungen über Funktionalgleichungen und ihre Anwendungen, Basel und Stuttgart 1961.

[2] - J. Kalmar et J. G. Mikusiński, Sur l'équation de translation, Studia Math. 12 (1951), pp. 112-116.

[3] U. T. Bödewadt, Zur Iteration reeller Funktionen, Math. Zeitschr. 49 (1944), pp. 497-516.

[4] M. Kuczma, On the Schröder equation, Rozprawy Mat. 34 (1963).

[5] A. Lundberg, On iterated functions with asymptotic conditions at a fixpoint, Arkiv för Matematik 5 (1963), pp. 193-206.

[6] H. Michel, Untersuchungen über stetige, monotone Iterationsgruppen reeller Funktionen ohne Differenzierbarkeitsvoraussetzungen, Publ. Math. Debrecen 9 (1962), pp. 13-46.

[7] G. Szekeres, Regular iteration of real and complex functions, Acta Math. 100 (1958), pp. 203-258.

[8] - On a theorem of Paul Lévy, A Magyar Tud. Akad. Mat. Kut. Int. Közl., A Sorozat, 5 (1960), pp. 277-282.

[9] M. Ward and F. B. Fuller, The contimuous iteration of real functions, Bull. Amer. Math. Soc. 42 (1936), pp. 393-396.

Regu par la Rédaction le 30.6.1965 